Orthopäde 2008 · 37:251-256

DOI 10.1007/s00132-008-1197-5

Online publiziert: 6. Februar 2008

(c) Springer Medizin Verlag 2008

\section{Redaktion}

V. Ewerbeck, Heidelberg

\author{
M. Janke ${ }^{1,3} \cdot$ M. Börner $^{2} \cdot$ E. Mueller-Garamvölgyi ${ }^{3} \cdot$ R. Hess ${ }^{1}$ \\ ${ }^{1}$ Spital STS AG Thun, Schweiz \\ ${ }^{2}$ Kantonsspital, Olten, Schweiz \\ ${ }^{3}$ Inselspital, Bern, Schweiz
}

\title{
56-jährige Patientin mit pathologischer subka- pitaler Humerusfraktur bei Morbus Gaucher
}

\section{Diagnostik}

Bei gutem Allgemein- und Ernährungszustand war die körperliche Untersuchung bis auf die schmerzhafte linke Schulter unauffällig. Das Routinelabor wies lediglich eine leichte Panzytopenie auf.

- Röntgen a.-p./Neer: pathologische subkapitale Humerusfraktur mit gekammerten osteolytischen Herden, zarter Kortikalis und leichter Osteopenie (• Abb. 1). Aufgrund des osteolytischen Röntgenbefunds und der Panzytopenie stand zunächst der Verdacht auf ein malignes Geschehens im Vordergrund, welcher vor Frakturversorgung abgeklärt werden musste.

- Ganzkörperszintigraphie: Minderanreicherung im Humerus, distalen Femur und der proximalen Tibia beidseits,

Abb. $1 \Delta$ Röntgen links Schulter a.-p./Neer
- MRT Oberarm links: diffus infiltrativer Prozess im gesamten Mark des Humerus mit partieller Ausdünnung der Kortikalis ohne Weichteilbeteiligung (• Abb. 2),

- MRT Femur beidseits: diffuse Knochenmarkalterationen sowie Erlenmeyer-Kolben-förmige Metaphysen beidseits, osteonekroseverdächtiger Befund links (• Abb. 3),

- CT Abdomen: Hepatosplenomegalie von $21 / 18 \mathrm{~cm}$ (Norm $\leq 15 / 12 \mathrm{~cm}$ ),

- CT-Thorax, Schilddrüsen-/Mammasonographie/gynäkologisches

Konsilium: ohne pathologische $\mathrm{Be}$ funde,

- Erweiterte Labordiagnostik: Retikulozyten und Ferritin erhöht; Transferrinsättigung erniedrigt, alle anderen Werte im Normbereich,

- Die Knochenmarkbiopsie (Beckenkamm) zeigte eine Verdrängung des hämatopoitischen Parenchyms mit Infiltration der Markräume durch sog. „Gaucher-Zellen“ (lipidspeichernde Makrophagen) mit der charakteristischen PAS-positiven „zerknittert“ fibrillären Struktur des Zytoplasmas (• Abb.4).
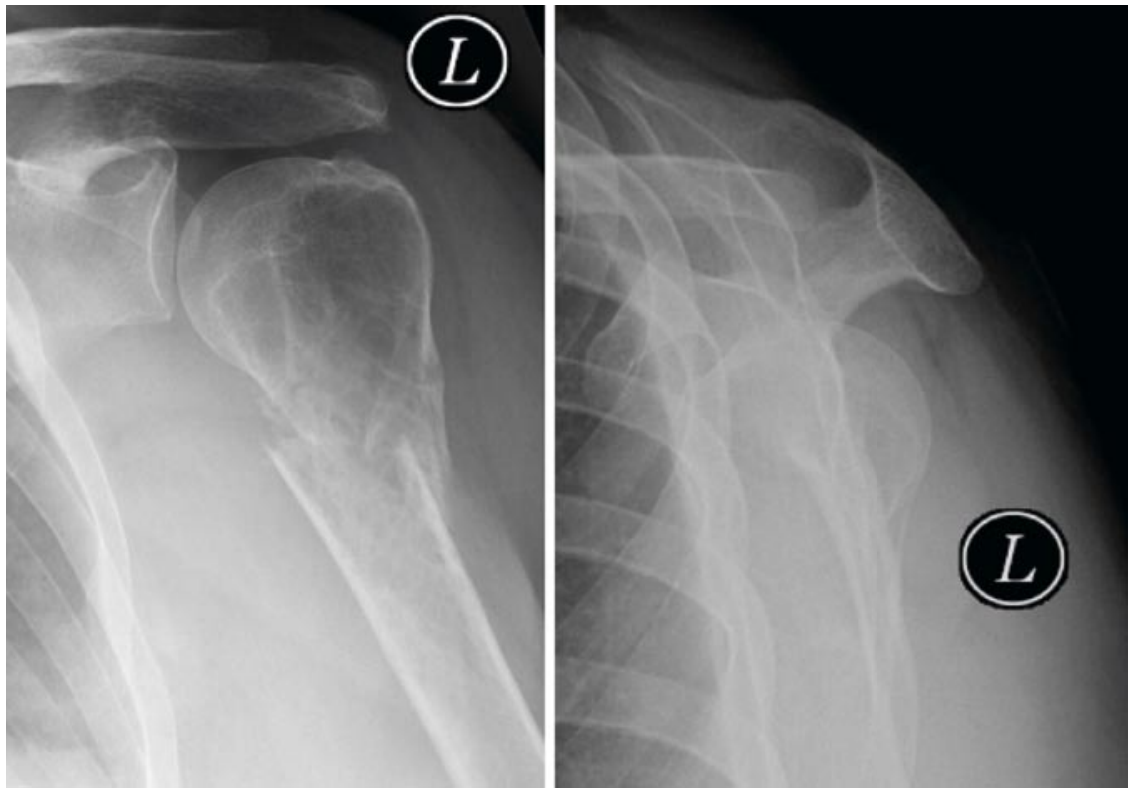


\section{Kasuistiken}
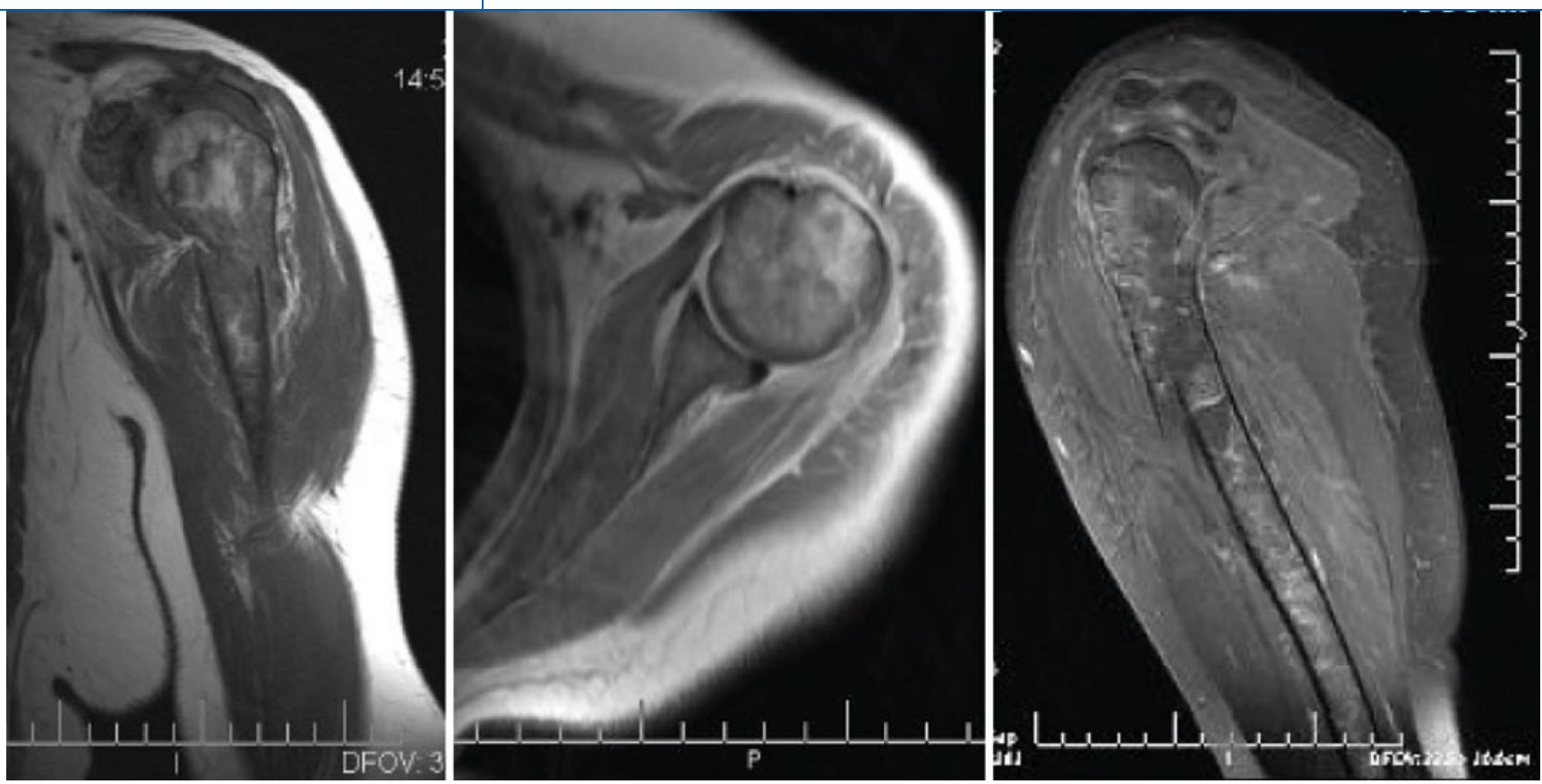

Abb. $2 \Delta$ MRT linker Oberarm
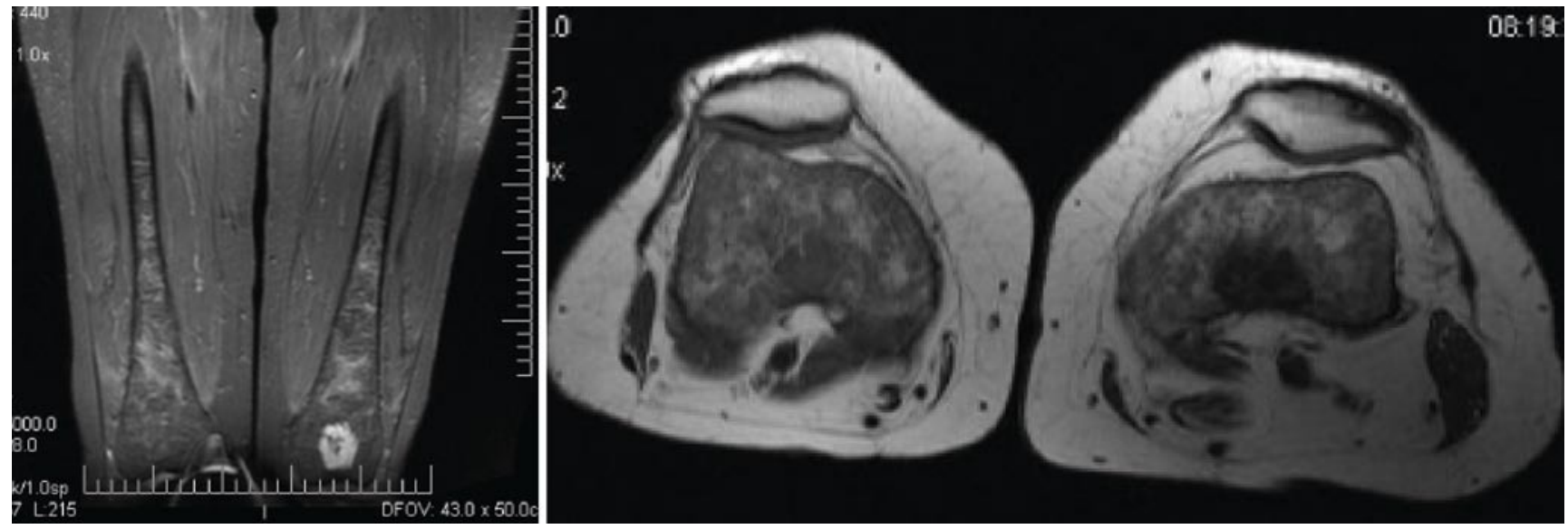

Abb. $3 \triangle$ MRT Femur beidseits

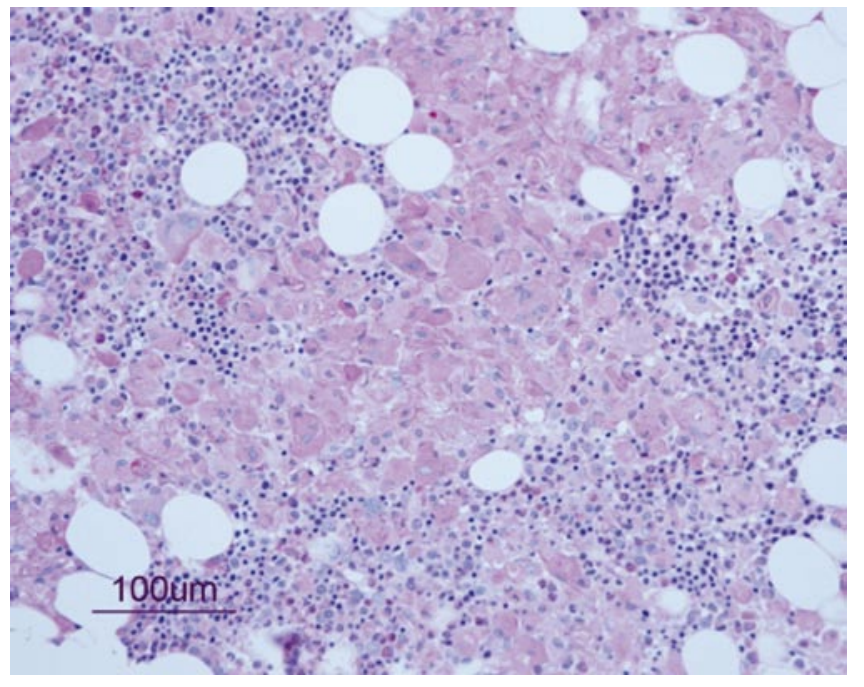

Abb. $4<$ PAS, Vergr. 40:1: Knochenmarkbiopsie
Zur Diagnosesicherung wurde die verringerte Aktivität der $\beta$-Glucosidase in Leukozyten bestimmt.

\section{Therapie und Verlauf}

Die operative Versorgung erfolgte mittels winkelstabiler Plattenosteosynthese. Die mit fettigem Gewebe ausgefüllte Metaphyse wurde auscurettiert und mit makroskopisch unauffälliger Beckenkammspongiosa aufgefüllt.

Bei geschwächter Knochenstruktur war eine vorsichtige aktiv assistierte Nachbehandlung für 6 Wochen geboten.

Radiologische Verlaufskontrollen bestätigten korrekte Stellungsverhältnisse und eine Konsolidation der Fraktur nach 
Hier steht eine Anzeige.

算 Springer 


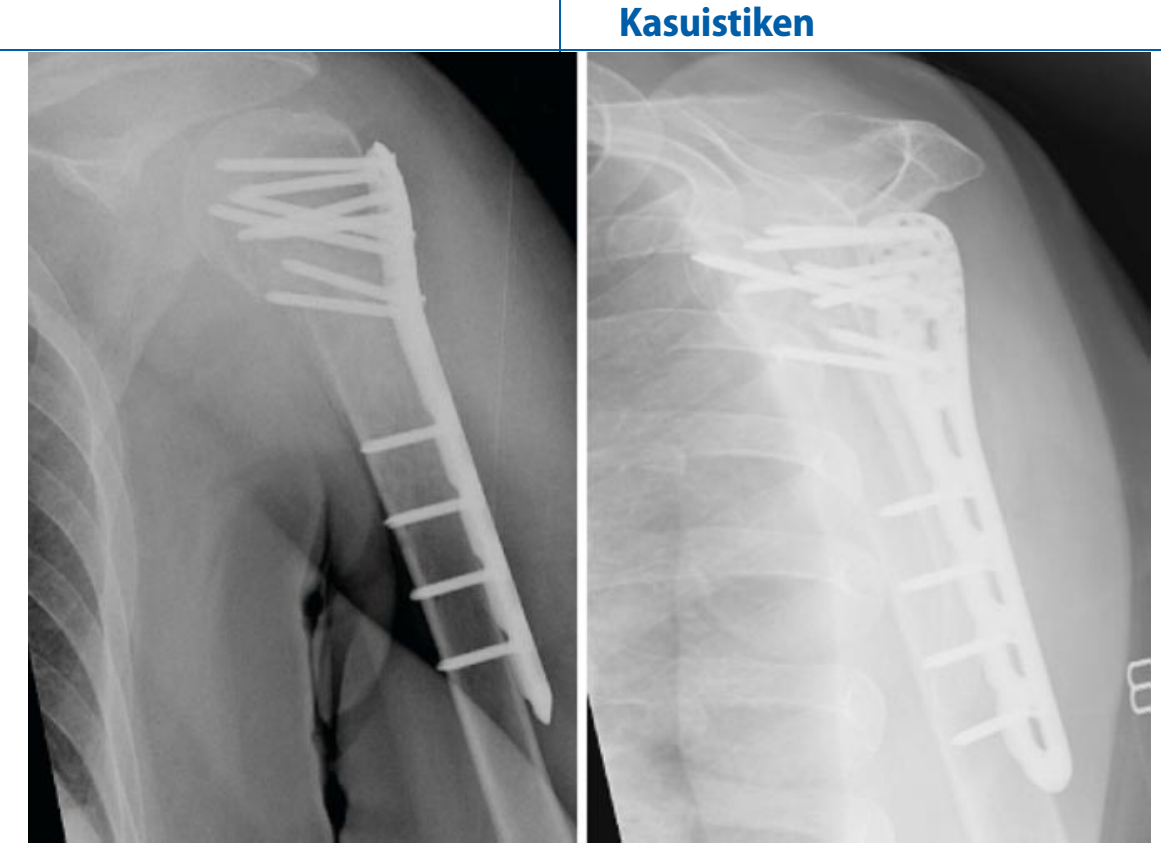

Abb. 5 A Postoperative Rx-Kontrolle

ca. 12 Wochen (• Abb. 5). Mit einer Elevation von $130^{\circ}$ und einer Innen-/Außenrotation von $\mathrm{Th} 8 / \mathrm{o} / 30^{\circ}$ nach 6 Monaten konnte die Schulterbeweglichkeit kontinuierlich verbessert werden.

Nach der Diagnosesicherung wurde die Enzymersatztherapie am universitären Zentrum für Stoffwechselerkrankungen eingeleitet.

\section{Diskussion}

\section{Ätiologie des Morbus Gaucher}

Die häufigste autosomal-rezessiv vererbte Sphingolipidose führt durch die verminderte Spaltung von Glucosylceramid zu dessen Akkumulation in Makrophagen des retikuloendothelialen Systems, v. a. in Milz, Leber und Knochenmark (GaucherSpeicherzellen) [1]. Die Inzidenz variiert zwischen 1:40.000 (Zentraleuropa) und 1:200o (z. B. Israel) $[1,7]$.

In Deutschland wird die Krankheit schätzungsweise nur bei $10-20 \%$ der Betroffenen diagnostiziert, was auf die häufig nur geringe Symptomatik und das unspezifische Erscheinungsbild des nicht neuronopathischen Typs zurückzuführen ist [4]. Dies erklärt auch die, wie auch in unserem Fall erst im fortgeschrittenen Alter erfolgte Manifestation und Diagnosestellung. Retrospektiv könnte auch beim Bruder der Patientin ein Morbus Gaucher vorgelegen haben.
Die Einteilung erfolgt in 3 Typen (• Infobox 1).

Die Diagnose wird durch Messung $\operatorname{der} \beta$-Glucosidase in Leukozyten gestellt und sollte durch den Nachweis des Gendefekts gesichert werden [4]. In unserem Fall wurde die genaue Art der Genmutation nicht bestimmt, da dieser Test von der Krankenkasse aufgrund geringer therawurde.

Milz und Leber können auf ein Vielfaches anwachsen und zu Milzinfarkt und abdominellen Schmerzen führen [4]. Eine Splenektomie sollte vermieden werden, da sich Knochenmanifestationen nachfolgend entscheidend verschlimmern können [3]. Anämie und Thrombozytopenie können im Verlauf zur Panzytopenie mit Thrombozyten $<20.000 / \mu l$ führen [4]. Am nachhaltigsten wird die Lebensqualität durch die Knochenmanifestationen v. a. im distalen Femur, proximaler Tibia und proximalen Humerus beeinträchtigt $[3,4]$.

Osteonekrosen führen durch Zelluntergang intramedullär zur Bildung unlöslicher „Calciumseife“, kortikal zu pathologischen Frakturen sowie teils akuten, hoch schmerzhaften „Knochenkrisen“, Fieber und Leukozytose. Osteopenie und Osteolysen bedingen eine erhöhte Frakturgefahr der großen Röhrenknochen und Wirbelkörper [3, 4]. peutischer Relevanz nicht übernommen
Radiologisches Merkmal des gestörten Knochenremodelling sind metaphysär Erlenmeyer-Kolben-förmige Auftreibungen mit ausgedünnter Kortikalis [3].

Die sensitivste Technik zur Dokumentation der Knochenveränderungen und zur Einstufung des Schweregrades ist die MRT [3, 5]. Differentialdiagnostisch lassen sich die MRT-Veränderungen von Metastasen, Tumoren, Osteomyelitis, Knocheninfakten oder malignen Erkrankungen des myelolymphatischen Formenkreises aufgrund der unterschiedlichen $\mathrm{Si}$ gnalintensität in der T2-Wichtung unterscheiden. Während die übrigen Erkrankungen zu einer Signalverstärkung in der T2-Wichtung führen, kommt es beim Morbus Gaucher sowohl in der T1- als auch der T2-Wichtung zu einer meist inhomogenen Signalverminderung mit sog. „Salz-und-Pfeffer-Mustern“. In die Überlegungen muss einbezogen werden, dass sehr aktive Formen gegenüber der T1Wichtung auch relativ hyperintens erscheinen können. Sowohl Knocheninfakte als auch aseptische Osteomyelitis zählen $\mathrm{zu}$ den Komplikation bei schweren Formen des Morbus Gaucher, womit diese gesondert betrachtet werden müssen $[2,3,5]$.

In der CT kann über die Bestimmung der Kortikalisdicke der Schweregrad festgelegt werden $[3,5]$. Die aufwendigste, aber als einzige quantitative Methode ist die MR-Messung des Fettanteils im Knochenmark QCSI (quantitatives chemisches Shift-Imaging [2, 4]).

Entscheidend für den langfristigen Verlauf ist die lebenslange Enzymersatztherapie mittels gentechnisch gewonnenem Enzym (Imiglucerase, Cerezyme), die zum Rückgang der Symptome und damit zur Wiederherstellung der Lebensqualität führt [4]. Die notwendige Enzymdosis orientiert sich stark an den ossären Veränderungen ( $\bullet$ Infobox 2) und kann verlaufsabhängig nach 6 Monaten angepasst werden [3, 4].

Bei der nicht-neuronopathischen Verlaufsform ist nach ca. 6 Monaten ein Rückgang der hämatologischen Veränderungen und der Leber-/Milzgröße, sowie nach 2 Jahren meist eine Normalisierung zu verzeichnen [4].

Die Knochenheilung wird durch Beeinträchtigung des Remodellingprozesses 
Infobox 1

\section{Einteilung des Morbus Gaucher}

- Nicht-neuronopathischer Typ (früher Typ I): chronischer Verlauf mit Hepatosplenomegalie, Knochenbefall und hämatologische Veränderungen; keine neurologischen Symptome; klinische Manifestation von der frühen Kindheit bis ins Erwachsenenalter.

- Akuter neuronopathischer Typ (früher Typ II): schwere neurologische Komplikationen; meist in den ersten 2 Lebensjahren letal endend.

- Chronisch neuronopathischer Typ (früher Typ III): mildere neurologische Symptome; geringere Progredienz und spätere Manifestation.

und durch eine verstärkte Osteopenie verzögert. Knochenveränderungen lassen sich im MRT frühestens nach 2-3 Jahren nachweisen, bedürfen bei schweren Komplikationen mindestens 3-4 Jahre einer hohen Dosis und eine Reduktion darf erst erfolgen, wenn die Besserung durch bildgebende Verfahren belegt ist $[3,4]$. Bei den neuronopathischen Verlaufsformen führt die Enzymsubstitution lediglich zur Besserung der nicht-neurologischen Komplikationen [4, 6]. Bei starkem Knochenbefall können zusätzlich Osteoklastenhemmstoffe (Pamidronate/Alendronate) gegeben werden, deren Einsatz aber bisher nicht in randomisierten Studien belegt ist $[3,4]$.

Bei fortgeschrittenen Verläufen sind prothetische und rekonstruktive Maßnahmen notwendig. Es empfiehlt sich eine begleitende Physiotherapie mit leichter körperlicher Belastung. Ein Ansatz zur Heilung des Morbus Gaucher ist die Gentherapie (Versuchsstadium), [3, 4].

Das Therapiemonitoring erfolgt 3-, später 6- bis 12-monatlich laborchemisch über die Chitotriosidase (korreliert mit dem gespeicherten Glucocerebrosid) und jährlich radiologisch mittels MRT (Veränderungen der unteren Extremität/LWS), $[2,3,4]$.

\section{Besonderheiten bei operativen Eingriffen}

Durch eine erhöhte Infektgefahr (grampositive Erreger), wahrscheinlich verursacht durch eine Makrophageninkompe-
Infobox 2

\section{Notwendige Dosierung der}

Enzymersatztherapie

- $60 \mathrm{IE} / \mathrm{kg} / 2$ Wochen: bei schweren Skelettkomplikationen, massiver Hepato-/Splenomegalie, Panzytopenie,

- 30-40 IE/kg/2 Wochen: bei deutlicher Hepatosplenomegalie/Panzytopenie und leichten Skelettveränderungen,

- $20 \mathrm{IE} / \mathrm{kg} / 2$ Wochen: Hepatosplenomegalie (Hypersplenismus) ohne wesentliche Knochenveränderungen.

tenz, empfiehlt sich die präoperative Enzymersatztherapie und prophylaktische Antibiotikatherapie ( $\geq_{4-7}$ Tage postoperativ [3]).

Bei erhöhter Blutungsneigung durch Thrombozytopenie/Schäden am hepatischen prothrombinbildenden System sollte auf Antikoagulantien möglichst verzichtet werden [3]. Bei der geschwächten Knochenstruktur sind winkelstabile Implantate empfehlenswert und autologe Spongiosaplastik eine Option.

\section{Fazit für die Praxis}

Bei Patienten mit pathologischen Frakturen, Osteolysen, Osteonekrosen oder Blutungsneigung unklarer Ätiologie sollte an einen Morbus Gaucher gedacht werden. Diagnostisch beweisend ist die Messung der $\beta$-Glucocerebrosidase in Leukozyten. Hierdurch ergeben sich entscheidende Konsequenzen für die operative Therapie und durch eine lebenslange Enzymersatztherapie eine wirksame Behandlungsoption.

\section{Korrespondenzadresse}

Dr. M. Janke

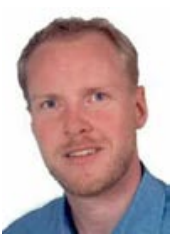

Spital STS AG Thun

Krankenhausstraße 12

CH-3600 Thun

Schweiz

markus.janke@web.de

Interessenkonflikt. Der korrespondierende Autor gibt an, dass kein Interessenkonflikt besteht.
Orthopäde 2008 · 37:251-256

DOI 10.1007/s00132-008-1197-5

(c) Springer Medizin Verlag 2008

M. Janke · M. Börner · E. Mueller-Garamvölgyi $\cdot$ R. Hess

56-jährige Patientin mit pathologischer subkapitaler Humerusfraktur bei Morbus Gaucher

\section{Zusammenfassung}

Wir berichten über eine Patientin mit pathologischer Humerusfraktur bei Erstdiagnose eines Morbus Gaucher, der häufigsten autosomal-rezessiv vererbten Lipidspeicherkrankheit. Sie führt häufig zu schweren Knochenkomplikationen mit Schmerzen, Osteonekrosen, Frakturen oder Gelenkeinbrüchen. Bei begründetem Verdacht sollte in Hinblick auf die therapeutischen Konsequenzen zur Diagnosesicherung die $\beta$-Glucocerebrosidase in Leukozyten bestimmt werden. Durch eine Enzymersatztherapie ist die Krankheit effektvoll behandelbar.

Schlüsselwörter

Morbus Gaucher · Pathologische Fraktur · Osteonekrosen - Enzymersatztherapie - Osteosynthese

\section{A 56-year-old patient with Gaucher's disease sustaining a pathologic subcapital fracture of the humerus}

\section{Abstract}

We present a case of a pathologic humerus fracture in a patient with the initial diagnosis of Gaucher's disease, which is the most frequent form of lipidosis transmitted as an autosomal recessive trait. It often results in orthopaedic complications with pain, osteonecrosis, fractures and joint infractions. If there is cause for suspicion, $\beta$-glucocerebrosidase in white blood cells should be measured because of the important consequences for treatment. Therapy with a modified enzyme is effective in managing the disease.

Keywords

Gaucher's disease - Pathologic fracture - Osteonecrosis - Enzyme therapy - Surgical treatment 


\title{
Literatur
}

\section{Hier steht eine Anzeige.}

\author{
算 Springer
}

1. Dürr EM, Geiß HC, Pontz BF, Parhofer KG (2004) 32jähriger Patient mit pathologischer Humerusfraktur, Splenomegalie und Thrombozytopenie. Internist 45: 455-460

2. Hermann G, Shapiro RS, Abdelwahab IF, Grabowski $G$ (1993) MR imaging in adults with Gaucher disease type I: evaluation of marrow involvement and disease activity. Skeletal Radiol 22: 247-251

3. Mankin HJ, Rosenthal DI, Xavier R (2001) Gaucher disease: new approches to ancient disease. J Bone Joint Surg Am 83: 748-763

4. Niederau C, Rolfes A (2000) Diagnose und Therapie des Morbus Gaucher. Med Klin 96: 32-39

5. Rosenthal DI, Mayo-Smith W, Goodsitt MM et al. (1989) Bone and bone marrow changes in Gaucher disease: Evaluation with quantitative CT. Radiologie 170: 143-146

6. Vellodi A, Bembi B, de Villemeur TB et al. (2001) Management of neuronopathic Gaucher disease: a European consensus. J Inherit Metab Dis 24: 319327

7. Zimran A, Gelbart T, Westwood B et al. (1991) High frequency of the Gaucher disease mutation at nucleotide 1226 among Ashkenazi jews. Am J Hum Genet 49: 855-859 